\title{
Estudo de interoperabilidade entre software BIM e softwares de análise energética de edificações (BES)
}

\author{
Interoperability study between software BIM and building \\ energy analysis software's (BES)
}

\author{
Fernanda Laura dos Santos Côrtes \\ Universidade Federal de Uberlândia | Uberlândia | Brasil | \\ fernandalauracortes@hotmail.com
}

Ana Carolina Fernandes Maciel

Universidade Federal de Uberlândia | Uberlândia | Brasil | anamaciel@ufu.br

\section{Resumo}

A utilização de softwares BIM (Building Information Modeling) e BES (Building Energy Simulation) facilitam as análises energéticas das edificações e tornam este processo preciso $e$ otimizado. No entanto, para que sejam obtidos resultados consistentes, faz-se necessário garantir a interoperabilidade entre esses. Nesta pesquisa, foi realizado um estudo de caso para

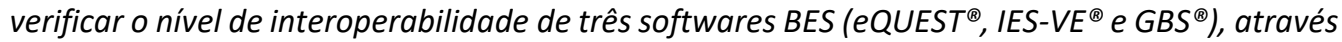
da exportação de um projeto modelo desenvolvido em software BIM (Revit $\left.{ }^{\circledR}\right)$. Para a avaliação do desempenho em relação a interoperabilidade entre os softwares, foram analisados cinco critérios e, com base nas análises destes e nos resultados encontrados, conclui-se que o eQUEST ${ }^{\circledR}$ apresentou as melhores condições de interoperabilidade dentre as opções analisadas.

Palavras-chave: Análise energética. BES. BIM. Interoperabilidade.

\begin{abstract}
The use of BIM (Building Information Modeling) and BES (Building Energy Simulation) software facilitate the energy analysis of buildings and make this process precise and optimized. However, to obtain consistent results, it is necessary to guarantee interoperability between software's. In this research, a case study was carried out to verify the performance of three BES software's (eQUEST ${ }^{\circledR}, I E S-V E^{\circledR}$ e GBS ${ }^{\circledR}$ ) trough exporting a sample modeled developed on BIM software (Revit $\left.{ }^{\circledR}\right)$. To evaluate the performance of the software's, five criteria were analyzed and, based on the analysis and on the results obtained, it is concluded that the eQUEST ${ }^{\circledR}$ software presented the best results in face of the analyzed criteria.
\end{abstract}

Keywords: Energy analysis. BES. BIM. Interoperability.

\section{INTRODUÇÃO}

A evolução dos programas computacionais para simulação da eficiência energética das edificações vem contribuindo de forma significativa para a consolidação desse

Como citar:

CÔRTES, F. L. dos S.; MACIEL , A. C. F. Estudo de interoperabilidade entre software BIM e softwares de análise energética de edificações (BES). In: SIMPÓsIO BRASILEIRO DE TECNOLOGIA DA INFORMAÇÃO E COMUNICAÇÃO NA CONSTRUÇÃO, 3., 2021, Uberlândia. Anais [...]. Porto Alegre: ANTAC, 2021. p. 1-13. Disponível em:

https://eventos.antac.org.br/index.php/sbtic/article/view/588. Acesso em: 3 ago. 2021. 
procedimento de análise. Essa tecnologia permite aos engenheiros e arquitetos, ainda na fase de projeto, analisarem as melhores soluções e materiais a serem empregados, para obter edificações com melhor desempenho térmico e melhores índices de eficiência energética.

No cenário atual, as tecnologias BIM (Building Information Modeling) e BES (Building Energy Simulation) são referências, sendo amplamente utilizadas para o estudo de construções sustentáveis devido à possibilidade da sobreposição de múltiplas informações dentro de um modelo, permitindo a análise detalhada e precisa da edificação.

Kota et al. [1] considera que o BIM não é apenas utilizado para criar o modelo da edificação, mas também como um repositório de informações de construção, que pode ser recuperado para realizar análises diferentes em edificações, como as de consumo energético, luz natural, estimativa de custos e estrutural. Wong e Zhou [2] apontaram a utilização do BIM como um processo baseado em modelo de geração e gerenciamento de dados de construção, coordenados e consistentes durante o ciclo de vida do projeto, que aprimoram o desempenho energético da edificação e facilitam o cumprimento das metas de sustentabilidade.

Para executar uma análise de desempenho energético em software's BES, de forma a obter resultados relevantes, é necessário garantir a interoperabilidade entre um modelo projetado em BIM e os programas de análise BES. Conforme conclui Chen, et al. [3], a produtividade no mundo BIM sofrerá com a falta de plataformas ou softwares compatíveis e, a interoperabilidade entre softwares configura a principal melhoria do mercado.

"A interoperabilidade é a capacidade de dois ou mais sistemas trocar informações e utilizar as informações trocadas" [4]. Em outras palavras, a interoperabilidade é a relação que os softwares possuem de transferência de dados, portanto, dizer que eles possuem boa interoperabilidade significa dizer que os dados e configurações feitas em um software conseguem ser transmitidos para outro sem grandes perdas. Considerando que os programas geram arquivos em variados formatos (.rvt, .gbXML, .inp), ao transferir as informações entre um e outro, podem ocorrem falhas na transferência de dados.

Diante disso, esta pesquisa teve como objetivo analisar a interoperabilidade entre um software BIM e softwares BES para, então, concluir quais tipos de informações estão sendo perdidas durante a transferência e, também, qual dos softwares possui melhor desempenho nesse quesito.

\section{METODOLOGIA}

Este trabalho foi realizado através de um estudo de caso, utilizando como base a metodologia adotada no artigo "Case studies for the evaluation of interoperability between a BIM based architectural model and building performance analysis programs" [5]. Assim como no artigo de MOON, H.J. et al., por meio de um projeto 
básico, modelado em software BIM e exportado para software's BES, são verificados diversos itens, no software de chegada (BES), para analisar a interoperabilidade entre o software de origem (BIM) e os de chegada (BES), sendo possível assim, identificar as vantagens ou falhas relacionas à interoperabilidade.

Para as análises de interoperabilidade, foram utilizados os softwares BES: Green Building Studio $^{\circledR}$ (versão 2021.99.8.170), IES-VE ${ }^{\circledR}$ (versão 2019) e eQUEST ${ }^{\circledR}$ (versão 3.65.7175). A modelagem da edificação foi realizada no software BIM, Revit ${ }^{\circledR}$ (versões 2020 e 2021). O projeto utilizado trata-se de um edifício de 2 pavimentos com $200 \mathrm{~m}^{2}$, composto por 1 saguão e 2 banheiros, no primeiro pavimento, e um escritório no segundo pavimento (Figuras 1 e 2).

Figura 1: Modelo 3D da edificação

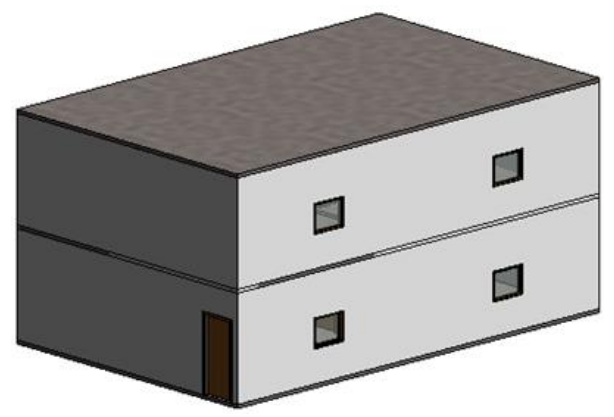

Fonte: os autores.

Figura 2: Planta baixa do primeiro e segundo pavimento, respectivamente
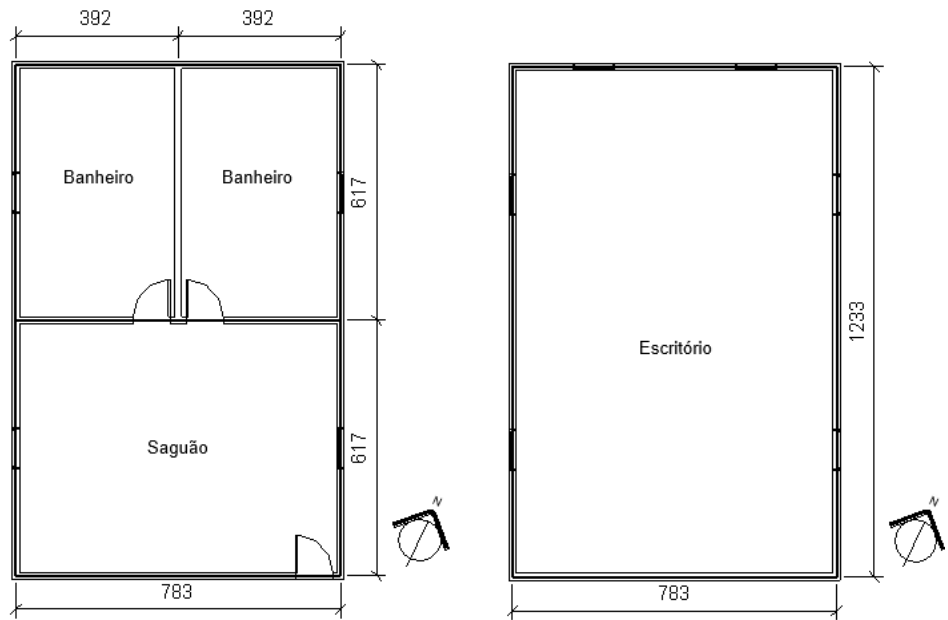

Fonte: os autores.

A transferência de dados para o estudo de interoperabilidade foi realizada de 3 maneiras (Figura 3): 
Figura 1: Métodos de transferência de dados

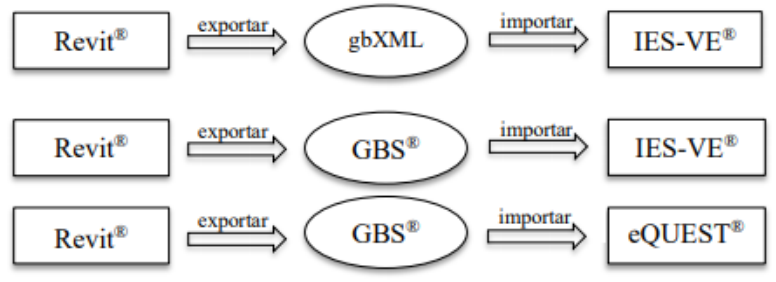

Fonte: os autores.

O software IES-VE ${ }^{\circledast}$ importa arquivos do tipo .gbXML e a exportação do projeto para este formato pode ocorrer de duas maneiras (1 e 2). No método 1, faz-se a exportação diretamente do software Revit ${ }^{\circledR}$, enquanto, no método 2 , o projeto é, primeiramente, exportado para o $\mathrm{GBS}^{\circledR}$, e, em seguida, exportado no formato .gbXML para o IES-VE ${ }^{\circledR}$. Foram utilizados ambos os métodos com o objetivo de analisar se haveria diferenças na transferência de dados entre esses.

Já o software eQUEST ${ }^{\circledR}$ importa arquivos do tipo inp, o qual não é exportado pelo software Revit ${ }^{\circledast}$. Diante disso, faz-se necessário e exportação para o $\mathrm{GBS}^{\circledR}$ e, em seguida, exportar para o formato .inp.

Para a análise da transferência de dados foram estabelecidos cinco critérios de análise, sendo eles: Geometria, Composição do espaço, Composição da construção, Cargas internas/Cronograma de ocupação, Sistema HVAC.

Geometria: análise visual do modelo no software, para verificar se a geometria exportada/importada, condiz com a geometria do modelo de energia no Revit ${ }^{\circledR}$ (Figura 4).

Figura 4: Modelo de energia do software Revit $^{\circledR}$

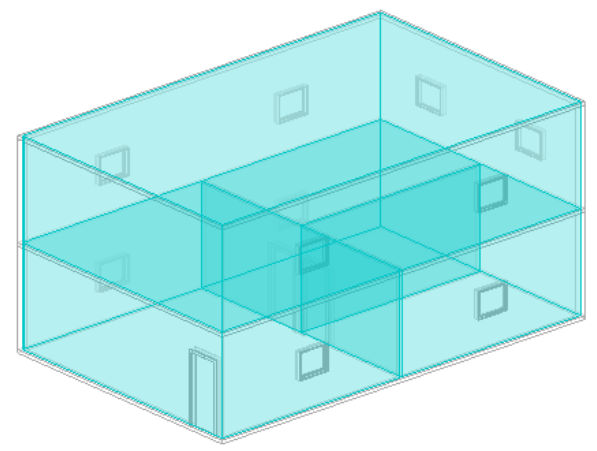

Fonte: os autores.

Composição do espaço: utilizada para verificar se as divisões de espaços e zonas foram transferidas adequadamente;

Composição da construção: para analisar se as informações de materiais, camadas, tipos de janelas, portas e demais elementos foram transferidos de maneira correta;

Cargas internas/Cronograma de ocupação: para verificar se houve a importação de informações relativas às cargas internas de iluminação, pessoas e equipamentos, além da agenda de ocupação de cada espaço; 
Sistema HVAC: para analisar a transferência de dados de condicionamento de ar.

\section{DADOS DE ENTRADA PARA O SOFTWARE REVIT ${ }^{\circledR}$ - SISTEMA DE}

AQUECIMENTO/RESFRIAMENTO

Para o sistema de aquecimento/resfriamento foi utilizado, entre os disponíveis no software, o "14SEER/0.9AFUE Split/Packaged Gas, <5.5 ton", visto que apresenta sistema split com gás, o qual é o mais utilizado na região e, concomitantemente, apresenta alta eficiência energética, de acordo com os parâmetros elucidados a seguir.

Para a análise do nível de eficiência energética de um aparelho de ar condicionado, é utilizado o parâmetro COP (Coefficient Of Performance) ou, como é denominado no Brasil, CEE (Coeficiente de Eficiência Energética). Esse parâmetro é utilizado pelo INMETRO [6], sendo os aparelhos de classe A os com melhor nível de desempenho, (Tabela 1).

Tabela 1: Classificação de eficiência

\begin{tabular}{ll}
\hline Classes & Coeficiente de Eficiência Energética (W/W) \\
\hline A & $3,23<$ CEE \\
\hline B & $3,02<$ CEE $<3,23$ \\
\hline C & $2,81<$ CEE $<3,02$ \\
\hline D & $2,60<$ CEE $<2,81$ \\
\hline
\end{tabular}

Fonte: INMETRO (2018).

No software Revit $^{\circledast}$, a eficiência do aparelho de ar condicionado é fornecida a partir do índice SEER (Seasonal Energy Efficiency Ratio). O SEER, entretanto, tem uma relação de equivalência com o CEE (Equação 1).

$C E E=\frac{S E E R}{1,2 \times 3,413}$

Diante disso, o aparelho utilizado possui SEER igual a 14, consequentemente, CEE de aproximadamente 3,42, pertencendo a Classe A na classificação do INMETRO.

\section{DADOS DE ENTRADA PARA O SOFTWARE REVIT ${ }^{\circledR}$ - LOCALIZAÇÃO}

Para a configuração de localização da edificação foi utilizado o Serviço de Mapeamento na Internet disponível no Revit ${ }^{\circledR}$. A localização definida para esta análise foi feita de forma aleatória visto que o estudo de interoperabilidade será realizado em uma edificação não existente, sendo especificado somente a cidade de Uberlândia com latitude $-18.9164^{\circ}$ e longitude $-48.25734^{\circ}$.

Com base na normativa internacional ASHRAE Standard 90.1: 2016 [7], que considera como parâmetro a localização do aeroporto de cada cidade para fins de análises energéticas, a estação utilizada foi a 837681 (Figura 5), a mais próxima do aeroporto de Uberlândia-MG. 
Figura 5: Localização e Estação Meteorológica - 837681

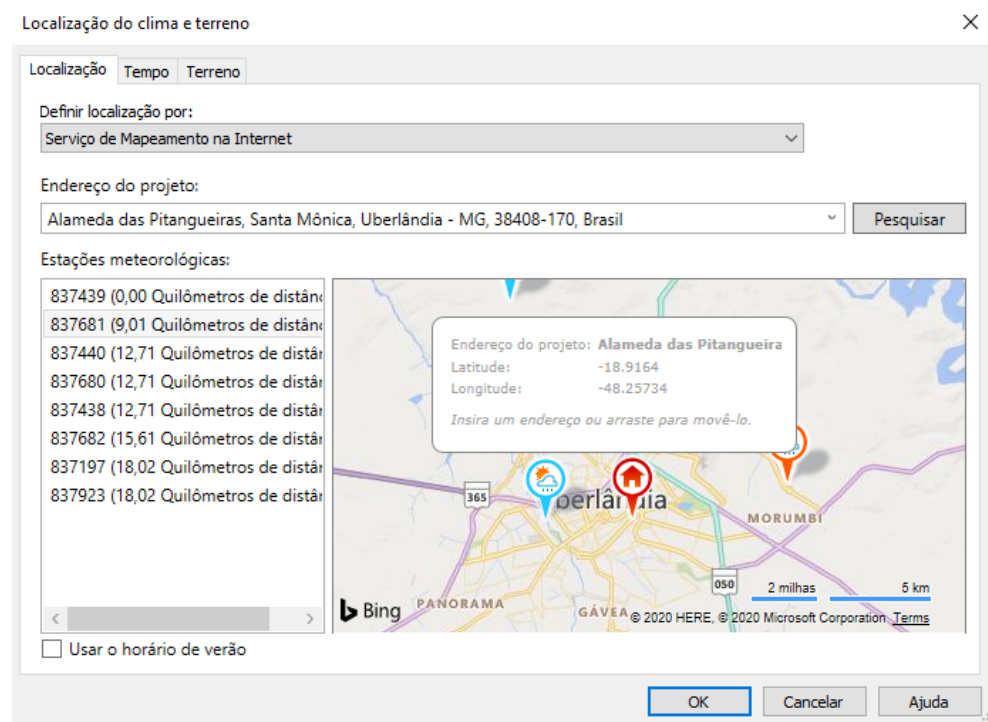

Fonte: os autores.

A localização da construção é de grande importância para a realização do estudo de eficiência energética visto que, a partir dela, são obtidas as temperaturas utilizadas no estudo.

Para validar os dados fornecidos pela Estação Meteorológica 837681, foi realizada uma comparação entre as temperaturas fornecidas pelo software Revit $^{\circledR}$ e as temperaturas disponíveis no Inmet (Instituto Nacional de Meteorologia) [8] (Figura 6). O Inmet utiliza como base de dados as temperaturas obtidas na estação $A 507$, enquanto o Revit ${ }^{\circledR}$ utiliza a estação 837681, diante disso, é esperado que haja pequena variação de temperatura.

Figura 6: Gráfico das temperaturas médias mensais da Estação Meteorológica A507 (Inmet) e Estação Meteorológica 837681 (Revit ${ }^{\oplus}$ )

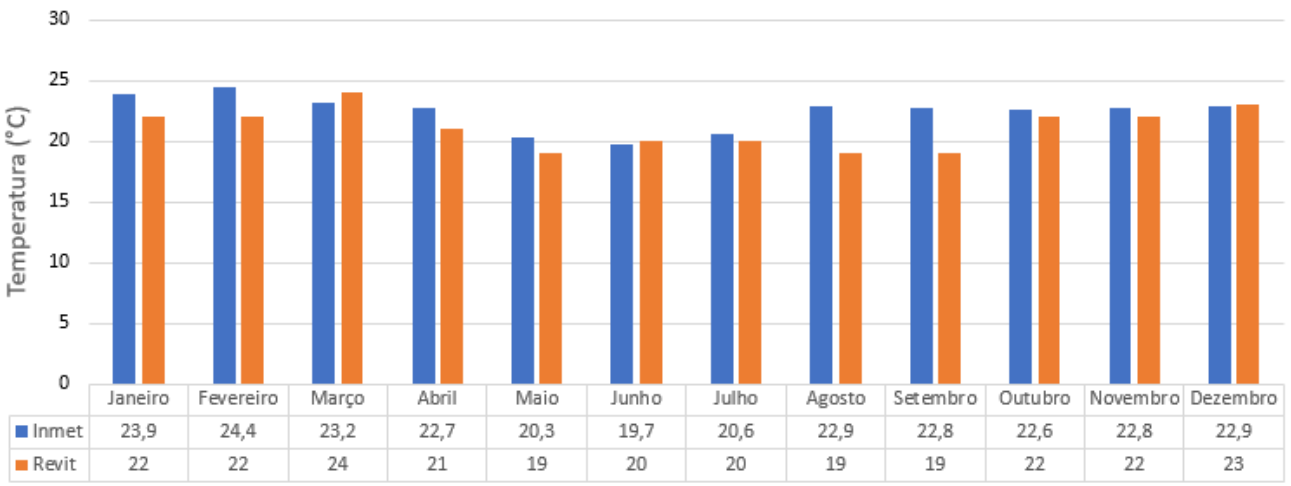

Fonte: os autores.

Por meio da comparação dos dados do Inmet com os dados de bulbo úmido do Revit ${ }^{\circledR}$, obteve-se a variação de temperatura apresentada abaixo (Tabela 2). 
Tabela 2: Variação da temperatura utilizada no software Revit $^{\circledR}$ e a obtida pelo Inmet

\begin{tabular}{lll}
\hline Mês & Graus $\left({ }^{\circ} \mathrm{C}\right)$ & Percentual (\%) \\
\hline Janeiro & 1,9 & 7,9 \\
\hline Fevereiro & 2,4 & 9,8 \\
\hline Março & 0,8 & 3,6 \\
\hline Abril & 1,7 & 7,5 \\
\hline Maio & 1,3 & 6,6 \\
\hline Junho & 0,3 & 1,6 \\
\hline Julho & 0,6 & 3,1 \\
\hline Agosto & 3,9 & 17,2 \\
\hline Setembro & 3,8 & 16,5 \\
\hline Outubro & 0,6 & 2,6 \\
\hline Novembro & 0,8 & 3,6 \\
\hline Dezembro & 0,1 & 0,5 \\
\hline Média & 1,5 & 6,7
\end{tabular}

Fonte: os autores.

Diante disso, verifica-se a existência de uma variação média de 6,7\% entre os dados, podendo-se considerar aceitável os dados de temperatura fornecidos pelo software Revit ${ }^{\circ}$.

\section{DADOS DE ENTRADA PARA O SOFTWARE REVIT ${ }^{\circledR}$ - ESPAÇOS}

Considerando a edificação analisada como um escritório, a classificação do edifício no software é "Escritório" (Figura 7).

\section{Figura 7: Configuração espaço "Escritório" no software Revit ${ }^{\circledR}$}

\begin{tabular}{|c|c|c|c|}
\hline \multirow{7}{*}{$\begin{array}{l}\text { Academia de Ginástica } \\
\text { Arena esportiva } \\
\text { Biblioteca } \\
\text { Centro de Convenções } \\
\text { Correios } \\
\text { Delegacia de Polícia } \\
\text { Depósito } \\
\text { Dormitório/Alojamento } \\
\text { Edificio religioso } \\
\text { Escola ou Universidade }\end{array}$} & \multirow[t]{7}{*}{$\wedge$} & Parâmetro & Valor \\
\hline & & Análise da energia & $\hat{\imath}$ \\
\hline & & Área por pessoa & $28.571 \mathrm{~m}^{2}$ \\
\hline & & Ganho de calor sensível por pessoa & $73.27 \mathrm{~W}$ \\
\hline & & Ganho de calor latente por pessoa & $58.61 \mathrm{~W}$ \\
\hline & & Densidade de carga de iluminação & $10.76 \mathrm{~W} / \mathrm{m}^{2}$ \\
\hline & & Densidade de carga de potência & $13.99 \mathrm{~W} / \mathrm{m}^{2}$ \\
\hline Escritório & & Contribuição de iluminação do plenum & $20.0000 \%$ \\
\hline $\begin{array}{l}\text { Fórum Judicial } \\
\text { Garagem }\end{array}$ & & Tabela de ocupação & Ocupação de escritório comum - 08:00 \\
\hline Ginásio & & Tabela de iluminação & lluminação do escritório - 06:00 a 23:00 \\
\hline $\begin{array}{l}\text { Hospital ou Posto de Saúde } \\
\text { Hotel }\end{array}$ & & Tabela de potência & lluminação do escritório - $06: 00$ a 23:00 \\
\hline Instalação automotiva & & Ar exterior por pessoa & $2.36 \mathrm{~L} / \mathrm{s}$ \\
\hline Manufatura & & Ar exterior por área & $0.30 \mathrm{~L} /\left(\mathrm{s} \cdot \mathrm{m}^{2}\right)$ \\
\hline $\begin{array}{l}\text { Motel } \\
\text { Multifamiliar }\end{array}$ & & Alterações do ar por hora & 0.000000 \\
\hline Museu & & Método de ar externo & por Pessoa e por Area \\
\hline Penitenciária & & Horário de abertura & $07: 00$ \\
\hline $\begin{array}{l}\text { Preteitura } \\
\text { Quartel de Bombeiros }\end{array}$ & & Horário de fechamento & $18: 00$ \\
\hline Restaurante de refeições rápidas & & Ponto definido de resfriamento sem ocu & $27.78^{\circ} \mathrm{C}$ \\
\hline
\end{tabular}

Fonte: os autores.

Foram utilizados 3 tipos de espaços para as análises, sendo eles: saguão, banheiros e escritório fechado (Figuras 8, 9 e 10). 
Figura 8: Configuração espaço "Saguão" no software Revit ${ }^{\circledR}$

\begin{tabular}{l}
\hline Quarto de estudos \\
Quarto de Paciente - Hospital/Posto de Saúde \\
Quartos de dormir - Delegacia/Quartel de Bombeiros \\
Recepção/Sala de Espera - Hotel \\
Recepção/Sala de Espera - Motel \\
Recepça/Sala de Espera - Transporte \\
Recuperação - Hospital/Posto de Saúde \\
Restauração - Museu \\
Reunião para conferências/Polivalente \\
Saquão \\
Saguão - Auditório \\
Saguão - Correios \\
Saguão - Edificio religioso \\
Saguão - Hotel \\
Saguão - Sala de Cinema \\
Saguão - Teatro \\
Saguão de Elevadores \\
Sala de audiência - Fórum Judicial \\
Sala de aula/Palestra/Treinamento \\
Sala de aula/Palestra/Treinamento - Penitenciária \\
Sala de equipamentos - Instalação Fabril \\
Sala de estar oública e de funcionários - Hospital/Pos
\end{tabular}

\begin{tabular}{|l|l|}
\hline \multicolumn{1}{|c|}{ Parâmetro } & \multicolumn{1}{|c|}{ Valor } \\
\hline Análise da energia & \\
\hline Área por pessoa & $0.667 \mathrm{~m}^{2}$ \\
\hline Ganho de calor sensível por pessoa & $73.27 \mathrm{~W}$ \\
\hline Ganho de calor latente por pessoa & $58.61 \mathrm{~W}$ \\
\hline Densidade de carga de iluminação & $13.99 \mathrm{~W} / \mathrm{m}^{2}$ \\
\hline Densidade de carga de potência & $5.81 \mathrm{~W} / \mathrm{m}^{2}$ \\
\hline Contribuição de iluminação do plenum & $20.0000 \%$ \\
\hline Tabela de ocupação & Ocupação de escritório comum - 08:00 \\
\hline Tabela de iluminação & lluminação do escritório - 06:00 a 23:00 \\
\hline Tabela de potência & lluminação do escritório - 06:00 a 23:00 \\
\hline Ar exterior por pessoa & $2.36 \mathrm{~L} / \mathrm{s}$ \\
\hline Ar exterior por área & $0.30 \mathrm{~L} /\left(\mathrm{s} \cdot \mathrm{m}^{2}\right)$ \\
\hline Alterações do ar por hora & 0.000000 \\
\hline Método de ar externo & por Pessoa e por Area \\
\hline
\end{tabular}

Fonte: os autores.

Figura 9: Configuração espaço "Banheiros" no software Revit ${ }^{\circledR}$

\begin{tabular}{|l|}
\hline <Construção> \\
Aeroporto - Entrada \\
Alojamentos - Dormitório \\
Alojamentos - Hotel \\
Alojamentos - Motel \\
Armazenagem ativa \\
Armazenagem ativa - Hospital/Posto de Saúde \\
Armazenagem inativa \\
Arquivo de cartões e catalogação - Biblioteca \\
Aéreo/Trem/Ônibus - Área de bagagens \\
Banheiros \\
Barbeiro e Salão de Beleza \\
Biblioteca - Audiovisual - Biblioteca audiovisual \\
Celas de confinamento - Fórum Judicial \\
Celas de confinamento - Penitenciária \\
Centro Cirúrgico - Hospital/Posto de Saúde \\
Compartimento de alta capacidade geral - Instalação \\
Compartimento de baixa capacidade geral - Instalaçấ \\
Corredor/Transição \\
Corredor/Transição - Instalação Fabril \\
Corredores com pacientes aguardando exame - Hosf \\
Culto - Pílnito do
\end{tabular}

\begin{tabular}{|l|l|}
\hline \multicolumn{1}{|c|}{ Parâmetro } & \multicolumn{1}{|c|}{ Valor } \\
\hline Análise da energia & $10.000 \mathrm{~m}^{2}$ \\
\hline Área por pessoa & $73.27 \mathrm{~W}$ \\
\hline Ganho de calor sensível por pessoa & $58.61 \mathrm{~W}$ \\
\hline Ganho de calor latente por pessoa & $9.69 \mathrm{~W} / \mathrm{m}^{2}$ \\
\hline Densidade de carga de iluminação & $3.23 \mathrm{~W} / \mathrm{m}^{2}$ \\
\hline Densidade de carga de potência & $20.0000 \%$ \\
\hline Contribuição de iluminação do plenum \\
\hline Tabela de ocupação & Ocupação de escritório comum - 08:00 \\
\hline Tabela de iluminação & lluminação do escritório - 06:00 a 23:00 \\
\hline Tabela de potência & lluminação do escritório - 06:00 a 23:00 \\
\hline Ar exterior por pessoa & $0.00 \mathrm{~L} / \mathrm{s}$ \\
\hline Ar exterior por área & $0.00 \mathrm{~L} /\left(\mathrm{s} \cdot \mathrm{m}^{2}\right)$ \\
\hline Alterações do ar por hora & 2.000000 \\
\hline Método de ar externo & por Pessoa e por Area \\
\hline
\end{tabular}

Fonte: os autores.

Figura 10: Configuração espaço “Escritório - Fechado" no software Revit ${ }^{\circledR}$

\begin{tabular}{|l|}
\hline Culto - Púlpito do Coro - Religioso \\
Detalhado - Instalação Fabril \\
Elétrica/Mecânica \\
Emergência - Hospital/Posto de Saúde \\
Escadaria \\
Escadas - Inativa \\
Escritório - Aberto \\
\hline Escritório - Fechado \\
\hline Espaço para exibições - Centro de Convençóes \\
Exames/Tratamento - Hospital/Posto de Saúde \\
Exibição geral - Museu \\
Farmácia - Hospital/Posto de Saúde \\
Gabinete de Juízes - Fórum Judicial \\
Hospital - Berçário - Hospital/Posto de Saúde \\
Hospital/Radiologia - Hospital/Posto de Saúde \\
Hotel/Centro de Conferência - Conferência/Reunião \\
Laboratório - Escritório \\
Laboratório da Delegacia de Polícia - Delegacia/Quar1 \\
Lavanderia - Lavar - Hospital/Posto de Saúde \\
Lavanderia - Passar e Separar \\
Local de veículos do quartel de bombeiros - Delegacia \\
Material de aualidade - Deoósito
\end{tabular}

\begin{tabular}{|l|l|}
\hline \multicolumn{1}{|c|}{ Parâmetro } & \multicolumn{1}{|c|}{ Valor } \\
\hline Análise da energia & $20.000 \mathrm{~m}^{2}$ \\
\hline Área por pessoa & $73.27 \mathrm{~W}$ \\
\hline Ganho de calor sensível por pessoa & $58.61 \mathrm{~W}$ \\
\hline Ganho de calor latente por pessoa & $11.84 \mathrm{~W} / \mathrm{m}^{2}$ \\
\hline Densidade de carga de iluminação & $16.15 \mathrm{~W} / \mathrm{m}^{2}$ \\
\hline Densidade de carga de potência & $20.0000 \%$ \\
\hline Contribuição de iluminação do plenum \\
\hline Tabela de ocupação & Ocupação de escritório comum - 08:00 \\
\hline Tabela de iluminação & lluminação do escritório - 06:00 a 23:00 \\
\hline Tabela de potência & lluminação do escritório - 06:00 a 23:00 \\
\hline Ar exterior por pessoa & $2.36 \mathrm{~L} / \mathrm{s}$ \\
\hline Ar exterior por área & $0.30 \mathrm{~L} /\left(\mathrm{s} \cdot \mathrm{m}^{2}\right)$ \\
\hline Alterações do ar por hora & 0.000000 \\
\hline Método de ar externo & por Pessoa e por Area \\
\hline
\end{tabular}

Fonte: os autores. 
A norma ASHRAE Standard 90.1:2016 estabelece que os sistemas HVAC devem ser ativados/desativados conforme agenda de ocupação, ou seja, de acordo com os horários que a edificação está sendo utilizada. Para esse estudo foram utilizadas as agendas de ocupação padrão do Revit $^{\circledR}$, que são determinadas pelo tipo de espaço. Portanto, as agendas utilizadas foram as agendas padrão dos ambientes: Escritório Fechado, Saguão e Banheiros.

Após as configurações especificadas neste item, foi criado e, posteriormente, gerado o Modelo de Energia do projeto. Com a criação deste modelo faz-se possível a exportação do projeto para os demais softwares.

\section{RESULTADOS E DISCUSSÕES}

O teste de interoperabilidade inicial foi realizado com o Green Building Studio ${ }^{\circledR}$. De acordo com a Autodesk, mesma desenvolvedora do software Revit ${ }^{\circledR}, \mathrm{O}$ GBS ${ }^{\circledast}$ [9] é uma plataforma nas nuvens para eficiência energética. Desta forma, as configurações de geometria, materiais, agenda de ocupação, sistema HVAC, localização e todas as demais são verificadas no próprio Revit $^{\circledR}$, sendo assim, não é possível verificar transferência de dados diretamente no $\mathrm{GBS}^{\circledR}$. Apesar disso, é a partir dele que é realizada a conversão dos tipos de arquivos a serem utilizados, possibilitando a importação do modelo BIM, para os softwares IES-VE ${ }^{\circledR}$ e eQUEST ${ }^{\circledR}$. Diante disso, podese afirmar que $\mathrm{o} \mathrm{GBS}^{\circledast}$ é fundamental para a realização das análises, possibilitando a importação do modelo da construção para os softwares de análise.

O segundo software a ser analisado foi o eQUEST ${ }^{\circledR}$. Em relação a geometria, pode-se concluir que a transferência foi realizada corretamente, uma vez que não foram encontradas divergências de informações entre Revit $^{\circledR}$ e eQUEST $^{\circledR}$ (Figura 11).

Figura 11: Geometria do modelo no software Revit $^{\circledR} \times$ Geometria do modelo no software eQUEST ${ }^{\oplus}$, respectivamente
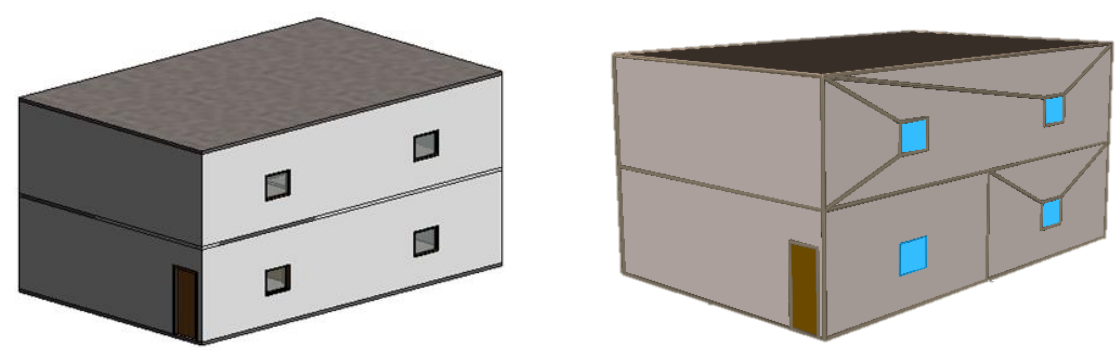

Fonte: os autores.

Quanto à composição do espaço, o eQUEST ${ }^{\circledR}$ identifica os 4 espaços criados e os agrupa em zonas de acordo com a configuração HVAC. As informações de cargas internas também se mostraram devidamente configuradas, juntamente com os cronogramas de ocupação de cada espaço.

Em relação à composição da construção, a transferência de dados não se mostrou eficiente, uma vez que os índices e materiais não foram importados de forma correta 
para o software. Quanto ao sistema HVAC, a nomenclatura do tipo de sistema de aquecimento/resfriamento configurado no software Revit $^{\circledR}$ diverge da nomenclatura utilizada no software eQUEST ${ }^{\circledR}$, entretanto, ao analisar suas características, conclui-se que consistem no mesmo tipo de sistema. No Quadro 1 são resumidos e especificados os parâmetros que foram e que não foram transferidos corretamente, ou seja, que tiveram problemas de interoperabilidade.

Quadro 1: Interoperabilidade do software eQUEST ${ }^{\circledR}$

\begin{tabular}{|c|c|c|}
\hline Critérios & Itens & eQUEST ${ }^{\circledR}$ \\
\hline \multirow{5}{*}{ Geometria } & Paredes & 0 \\
\hline & Portas & 0 \\
\hline & Janelas & 0 \\
\hline & Número de pavimentos & 0 \\
\hline & Laje & 0 \\
\hline \multirow{2}{*}{ Composição do espaço } & Zona & 0 \\
\hline & Espaço & 0 \\
\hline \multirow{6}{*}{ Composição da construção } & Espessura & $x$ \\
\hline & Condutividade térmica & $x$ \\
\hline & Calor específico & 0 \\
\hline & Rugosidade & $x$ \\
\hline & Densidade & 0 \\
\hline & Resistência térmica & $x$ \\
\hline \multirow{2}{*}{ Cargas internas/ Cronograma de ocupação } & Cargas internas & 0 \\
\hline & Cronograma de ocupação & 0 \\
\hline \multirow{2}{*}{ Sistema HVAC } & Tipo de sistema & 0 \\
\hline & Características térmicas do sistema & 0 \\
\hline
\end{tabular}

Fonte: os autores.

Nota: $\mathrm{O}=$ compatível; $\mathrm{X}=$ incompatível. Fonte: os autores.

$O$ terceiro software analisado foi o IES-VE ${ }^{\circledR}$. Em relação à geometria, pode-se concluir que a transferência foi realizada de forma correta (Figura 12). O IES-VE ${ }^{\circledR}$ identifica os 4 espaços criados e seu agrupamento em 3 zonas HVAC, tendo boa transferência de dados na composição do espaço. Já em relação à composição da construção, assim como no eQUEST ${ }^{\oplus}$, a interoperabilidade se mostrou falha, não sendo transferidos os materiais e índices térmicos como especificados no Revit ${ }^{\circledR}$.

Figura 12: Geometria do modelo no software Revit $^{\circledR} \times$ Geometria do modelo no software IES$\mathrm{VE}^{\circledR}$, respectivamente
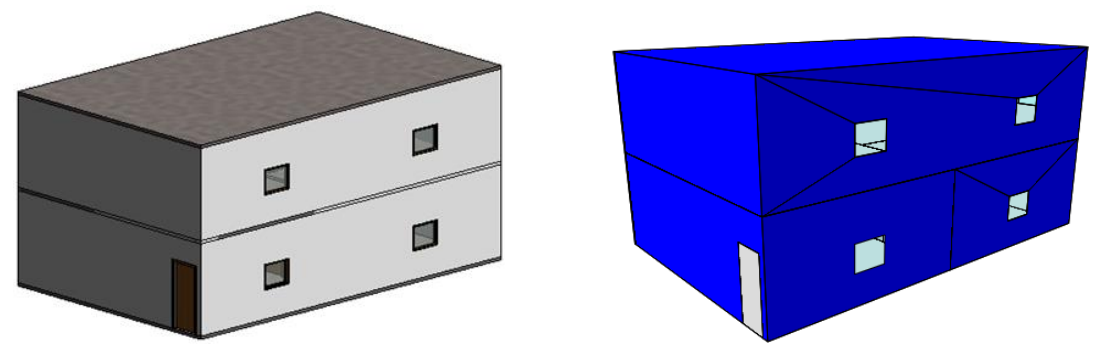

Fonte: os autores. 
O software recebeu a importação das informações de cargas internas da edificação, apesar da alteração nas nomenclaturas. Também não importou corretamente a agenda de ocupação dos espaços de acordo com o tipo de ambiente. O sistema HVAC importado assemelha-se ao configurado no Revit $^{\circledR}$, sendo também um sistema Split com ventilação mecânica e resfriamento, entretanto, as especificações relativas à eficiência energética dos aparelhos de ar condicionado não foram transferidas corretamente. No Quadro 2 são resumidos e especificados os parâmetros que foram e que não foram transferidos corretamente, ou seja, que tiveram problemas de interoperabilidade.

Quadro 2: Interoperabilidade do software IES-VE ${ }^{\circledR}$

\begin{tabular}{|c|c|c|}
\hline Critérios & Itens & IES-VE $^{\circledR}$ \\
\hline \multirow{5}{*}{ Geometria } & Paredes & 0 \\
\hline & Portas & 0 \\
\hline & Janelas & 0 \\
\hline & Número de pavimentos & o \\
\hline & Laje & o \\
\hline \multirow{2}{*}{ Composição do espaço } & Zona & o \\
\hline & Espaço & 0 \\
\hline \multirow{6}{*}{ Composição da construção } & Espessura & o \\
\hline & Condutividade térmica & $x$ \\
\hline & Calor específico & $x$ \\
\hline & Rugosidade & $x$ \\
\hline & Densidade & 0 \\
\hline & Resistência térmica & $\mathrm{x}$ \\
\hline \multirow{2}{*}{ Cargas internas/ Cronograma de ocupação } & Cargas internas & o \\
\hline & Cronograma de ocupação & $x$ \\
\hline \multirow{2}{*}{ Sistema HVAC } & Tipo de sistema & 0 \\
\hline & Características térmicas do sistema & $x$ \\
\hline
\end{tabular}

Fonte: os autores.

Nota: $\mathrm{O}=$ compatível; $\mathrm{X}$ = incompatível. Fonte: os autores.

De acordo com Porsani at al. [10], a falta de interoperabilidade BIM-BES é uma das lacunas existentes entre a digitalização e a área da construção. Como os softwares BES são a base dos certificados de desempenho energético, eles devem apresentar resultados precisos, de forma a garantir a confiança dos investidores no setor de eficiência energética.

Andrade e Ruschel [11] acreditam que a não existência de um padrão entre aplicativos para a descrição e classificação das propriedades dos componentes da construção dificulta a interoperabilidade dessas propriedades. Em concordância, Haagenrud [12] aponta a necessidade de um esforço internacional no sentido de se criar padrões para objetos a fim de melhor a classificação das propriedades dos componentes, contribuindo para melhorar a interoperabilidade. 
Atualmente, o sistema que mais se aproxima de uma padronização é o IFC (Industry Foudation Classes). O IFC é uma ferramenta que possui um formato de dados específico com a finalidade de permitir o intercâmbio de informações entre plataformas. Entretanto, o que se observa na prática, de acordo com Kiviniemi et al. [13], é que o uso de padrões IFC atende a requisitos para certas tarefas, deixando, contudo, que muitas outras tarefas não sejam suportadas por este formato. Além disso, os modelos IFC, apesar de apresentar boa interoperabilidade entre softwares BIM, não é recomendado para análises BES. De acordo com Queiróz [14], isso ocorre por esse formato de arquivo não ser destinado a essa finalidade, necessitando de programas auxiliares para conversão, tornando o processo mais trabalhoso e aumentando a chance de perdas de informações.

\section{CONCLUSÕES}

A pesquisa teve como objetivo analisar a interoperabilidade entre softwares BIM e BES por meio de um estudo de caso. Esta investigação permite a identificação de falhas recorrentes na transferência de informações entre softwares, possibilitando melhorias por parte das desenvolvedoras, como também orienta os profissionais da área de eficiência energética.

Com base nas análises efetuadas e nas considerações realizadas no decorrer do estudo é possível destacar que os dois softwares analisados, que possuem desenvolvedora diferente do software Revit $^{\circledR}$, apresentaram falhas na transmissão das informações e, portanto, não apresentaram interoperabilidade completa. Apesar disso, em alguns critérios analisados, a transferência de dados ocorreu de maneira satisfatória.

Com base na comparação entre os Quadros 1 e 2 é possível concluir que o software eQUEST ${ }^{\circledR}$ apresentou melhor interoperabilidade no estudo realizado, visto que houve boa interoperabilidade em maior número de critérios quando comparado com o IES$V^{\circledR}{ }^{\circledR}$, além de apresentar interface mais amigável para a realização das análises.

Faz-se importante ressaltar que o estudo de caso realizado aborda uma edificação simples, com apenas 2 pavimentos e nível de detalhamento baixo. Desta forma, é esperado que, ao analisar projetos de maior complexidade, maior quantidade de problemas de interoperabilidade possa ser encontrada.

Assim como no estudo que embasou este, o software eQUEST ${ }^{\circledR}$ ainda se mostra o mais eficiente em relação à interoperabilidade. Em concordância com o estudo, percebese a necessidade de aprimoramento na transferência de dados entre softwares BIM e BES. Considerando a diferença de 10 anos existente entre um estudo e outro, eram esperados resultados mais positivos de interoperabilidade entre os softwares. Desta forma confirma-se a importância de melhorias a fim de obter análises de eficiência energética mais precisas e otimizadas. 


\section{REFERÊNCIAS}

[1] KOTA, S. et al. Building Information Modeling (BIM)-based daylighting simulation and analysis. Energy and Buildings, v. 81, p. 391-403. Elsevier B.V. 2014. DOI: http://dx.doi.org/10.1016/j.enbuild.2014.06.043

[2] WONG, J.; ZHOU, J. Enhancing environmental sustainability over building life cycles through green BIM: A review. Automation in Construction, v. 57, p.156 - 165. Elsevier B.V. 2015. DOI: http://dx.doi.org/10.1016\%2Fj.autcon.2015.06.003

[3] CHEN, S. et al. Investigation of interoperability between building information modelling (BIM) and building energy simulation (BES). International Review of Applied Sciences and Engineering, v. 9, p.137-144. 2018. DOI: http://dx.doi.org/10.1556/1848.2018.9.2.9

[4] OSELLO, A. et al. Architecture data and energy efficiency simulations: BIM and interoperability standards. In: CONFERENCE OF INTERNATIONAL BUILDING PERFORMANCE SIMULATION ASSOCIATION, 12, Sidney, Australia. Proceedings [...]. Sydney, Australia: 2011. p.2210-2217.

[5] MOON, H.J. et al. Case studies for the evaluation of interoperability between a BIM based architectural model and building performance analysis programs. In: CONFERENCE OF INTERNATIONAL BUILDING PERFORMANCE SIMULATION ASSOCIATION, 12, Sidney, Australia. Proceedings [...]. Sydney, Australia: 2011. p.1521-1526.

[6] INSTITUTO NACIONAL DE METROLOGIA, NORMALIZAÇÃO E QUALIDADE INDUSTRIAL (INMETRO). Regulamento Técnico da Qualidade do Nível de Eficiência Energética de Edifícios Comerciais, de Serviços e Públicos. Anexo da Portaria INMETRO no 163/2009. 2009.

[7] AMERICAN NATIONAL STANDARDS INSTITUTE. NORRAE STANDARD 90-1: Energy Standard for Buildings Except Low-Rise Residential Buildings. Atlanta, 2013.

[8] INMET - Instituto Nacional de Metereologia. Tabelas de dados das estações. Disponível em: < http://www.inmet.gov.br>. Acesso em 13 de agosto de 2018.

[9] AUTODESK. Green Building Studio. Disponível em: < https://gbs.autodesk.com/GBS/>. Acesso em: 01/2020.

[10] PORSANI, G.B. et al. Interoperability between Building Information Modelling (BIM) and Building Energy Model (BEM). Applied Sciences, v.11, p.2167.2021.

[11] ANDRADE, M. L. V. X.; RUSCHEL. R. C. Interoperabilidade de aplicativos BIM usados em arquitetura por meio do formato IFC. Gestão \& Tecnologia de Projetos. v.4, p76-111. 2009.

[12] HAAGENRUD, S. et al. STANDINN Deliverable D15 IFC and IFD feasibility for innovative sustainable housing. EUROPA INNOVA, p.86. 2007.

[13] KIVINIEMI, A. Support for Building Elements in the IFC $2 \times 3$ Implementations based on 6th Certification Workshop Result. VTT Internal Report, 2007.

[14] QUEIRÓZ. G.R. Análise da interoperabilidade entre os programas computacionais Autodesk Revit e EnergyPlus para simulação térmica de edificações. Tese (Mestrado em Engenharia Civil) - Programa de Pós-Graduação em Engenharia Civil, Universidade Federal de Santa Maria. Santa Maria, p.181. 2016. 